\title{
Nuevas tecnologías y estudiantes chilenos de secundaria. Aportes a la discusión sobre la existencia de nuevos aprendices
}

\author{
New technologies and Chilean secondary students. Contributions to the discussion \\ on the existence of new apprentices \\ Novas tecnologias e estudantes chilenos do Ensino Secundário. \\ Contribuições para a discussão sobre a existência de novos aprendizados
}

\author{
Paulina A. Ruiz \\ Pontificia Universidad Católica de Chile, Facultad de Educación. Telf.: +56(2) 3545350. \\ Correo electrónico: pnruiz@uc.cl
}

\begin{abstract}
RESUMEN
Desde finales de la década del 2000 se comienza a criticar la propuesta sobre una nueva generación de jóvenes aprendices capaces de interactuar con las nuevas tecnologías como si fueran su lengua nativa. Se ha argumentado principalmente que falta investigación empírica que dé cuenta de diferentes tipos de uso de tecnologías y de estudiantes, así como de estudios cualitativos que incorporen sus percepciones y el análisis de distintos contextos de aprendizaje. Este estudio pretende aportar a esta discusión, analizando el uso que le dan jóvenes chilenos de educación secundaria a las nuevas tecnologías y sus percepciones sobre la contribución de ellas en el contexto escolar y extra-escolar. Se concluye que entre los estudiantes de la muestra existen escasos rasgos de los llamados nuevos aprendices y aparece una brecha perceptual importante entre distintos contextos, donde el aprendizaje se conceptualiza desde una mirada escolarizada.
\end{abstract}

Palabras clave: nuevos aprendices, nativos digitales, nuevas tecnologías, TIC, educación secundaria.

\begin{abstract}
Since late 2000, some reviewers have criticized the proposal on a new generation of young learners that can interact with new technologies like they were their native language. It has been argued mainly that there is a lack of empirical research which takes into account different types of technology use and kinds of students, as well as qualitative studies that incorporate their perceptions and analysis of different learning contexts. This study seeks to contribute to this discussion by analyzing the technology use of Chilean secondary students and their perceptions on the contribution of technologies inside and outside their schools. It is concluded that among students in the sample there are scarce traits of the called new apprentices and appears a perceptual gap between different contexts, where learning is conceptualized from a schooling perspective.
\end{abstract}

Key words: new apprentices, digital natives, new technologies, ICT, secondary education.

\section{RESUMO}

Desde o final de 2000, inicia-se a crítica da proposta de uma nova geração de jovens aprendizes capazes de interagir com as novas tecnologias como se estas fossem sua língua nativa. Tem-se argumentado, principalmente, que falta pesquisa empírica que dê conta de diferentes tipos de uso da tecnologia e de estudantes, bem como estudos qualitativos que incorporem suas percepções e análise de diferentes contextos de aprendizagem. Pretende-se contribuir para esta discussão, analisando o uso que os jovens chilenos da Educação Secundária dão às novas tecnologias e suas percepções da contribuição destas no contexto escolar e extraescolar. Conclui-se que, entre os estudantes da amostra, há poucos traços dos chamados novos aprendizes e aparece uma importante brecha perceptual entre diferentes contextos, de onde a aprendizagem é conceituada a partir de uma perspectiva escolarizada.

Palavras chave: novos aprendizes, nativos digitais, novas tecnologias, tic, educação secundária. 


\section{INTRODUCCIÓN}

A menudo como justificación al proceso de integración de Nuevas Tecnologías (NT o TIC) al contexto educativo se acude a los postulados de las teorías de nuevos aprendices o nativos digitales (TNA) (e.g. Prensky, 2001), las cuales postulan la existencia una nueva generación que se constituye como un grupo diferente a sus antecesores por su relación constante y sistemática con las NT. Se trata de una generación que desafía al mundo educativo a cambiar sus prácticas tradicionales a unas innovadoras, que incluyan la utilización de NT y que se adecuen a la nueva forma de aprender de ellos. La nueva generación sería consciente de las diferencias entre el uso de NT en el contexto escolar y el cotidiano, y serían capaces de orientarnos hacia los cambios esperados.

Sin embargo, desde finales de la década del 2000 se ha puesto en jaque la idea de la existencia generalizada de esta nueva generación de jóvenes aprendices (e.g. Bennett y Maton, 2010). Factores como el género, el nivel socioeconómico, los intereses personales, el acceso a las NT estarían explicando la existencia de distintos tipos de uso de NT y de jóvenes. Aun el avance en la recolección de evidencia, estos estudios se sitúan casi mayoritariamente en los mismos lugares donde nacieron estas teorías: países desarrollados y de habla inglesa y enfocados casi exclusivamente en contextos educativos de educación superior.

Aunque existen algunos estudios para educación escolar y en otros países (e.g Sánchez et al., 2010), queda mucho por hacer en otros contextos culturales. Este es el caso de Latinoamérica, donde es común en discursos, políticas y estudios el uso del término 'nativos digitales'. Como ejemplo, en Chile -en gran parte por sus altas tasas de acceso y uso de NT en comparación a otros países latinoamericanos y entre su población joven de 5 a 24 años (ITU, 2008 y 2010)- el lenguaje y planteamientos de las TNA han sido adoptados sin una base empírica propia y sólida. Esto ha impedido, por ejemplo, identificar perfiles variados de estudiantes y jóvenes que consideren variables como urbanidad/ruralidad o GSE bajos (e.g. IGD, 2009) o distintos espacios de aprendizaje con NT, dando a conocer una realidad tecnologizada homogénea que requiere de mayor investigación y análisis.

Este estudio pretende aportar a la discusión sobre nuevos aprendices, caracterizando a jóvenes chilenos de educación secundaria a partir del uso y frecuencia que les dan a las NT y las percepciones que tienen sobre su contribución en el contexto escolar y extra-escolar.

\section{REVISIÓN BIBLIOGRÁFICA}

\subsection{TEORÍAS SOBRE NUEVOS APRENDICES: POSIBILIDADES DE UNIR CONTEXTOS}

Desde finales de la década de los 90 surge una serie de informes y artículos que plantean la existencia de una nueva generación de jóvenes y aprendices, denominándolos de distintas maneras: 'Net generation' (Oblinger y Oblinger, 2005; Tapscott, 1998), 'Digital Natives' (Prensky, 2001a y 2001b), ‘Aprendices del nuevo milenio' (Pedró, 2006). Estas teorías postulan que la exposición a y el uso de NT de los jóvenes nacidos aproximadamente después de $1980^{1}$ produce que ellos manejen los códigos de estas tecnologías del

No hay acuerdo entre algunos investigadores sobre la fecha, incluso década exacta, por lo que 1980 sirve de referencia. 
mismo modo que un nativo maneja su lengua natal. La interacción que han tenido con ellas es novedosa y variada, por lo que redefine su forma de comunicarse, de pensar y de conocer, y por ende, de aprender. Se sienten cómodos con la tecnología, son capaces de usar variados y múltiples canales para acceder a información y comunicarse con otros, lo que se traduce en altos niveles de competencias en el uso de NT. En contraposición, generaciones anteriores se constituyen como otro grupo generacional, que al igual que un 'inmigrante', ha tenido que aprender el lenguaje de las NT con posterioridad (Prensky, 2001a).

Se trataría de una generación acostumbrada a la inmediatez y con la habilidad de aprender a través de información discontinua y variada; que aprende orientada por tareas (task-oriented) y que ejecuta sin problemas el multi-tasking (varias tareas al mismo tiempo); que prefiere los gráficos e imágenes (lenguaje multimedia) antes que el texto escrito; aprende en base al juego y diversión; y es capaz de gestionar su propio aprendizaje y trabajo colaborativo.

Dada estas características, se plantea que aparecen tensiones entre el contexto cotidiano de los nuevos aprendices y el contexto escolar, representado por profesoresinmigrantes digitales (Prensky, 2001a). Esta tensión también es postulada por teóricos hispanohablantes de la educomunicación, que algunos años antes que los anglosajones expusieron teorías sobre la brecha entre ambos contextos desde una perspectiva más cultural (Martín Barbero, 1996; Ferrés, 2000). Postulan que con la llegada de los medios masivos de comunicación surge una tensión entre la cultura de la escuela (identificada con la cultura del libro, la memoria, la racionalidad) con la cultura mediática del entorno (asociada a la cultura de la imagen y lo sensorial). Estos teóricos, al igual que aquellos que sostienen las TNA, postulan que el contexto escolar no incluye la realidad mediática/ tecnológica de sus educandos.

Aun existiendo esta brecha y tensión entre contextos, Prensky (2001a) plantea que los jóvenes tienen preferencias y estilos particulares de aprendizaje que serían capaces de comunicar y de traspasar al contexto escolar, pudiendo reinventar el colegio por sí mismos (Prensky, 2006). Esto debido en gran medida a la capacidad de la nueva generación de percibir las contradicciones entre sus prácticas con NT dentro y fuera del contexto escolar (Pedró, 2006), identificando aquellas que la escuela no incorpora y que serían positivas para ellos, "in part because they are things that the students have already mastered and use in their daily lives, and in part because they realize just how useful they can be" (Prensky, 2007: 41). Según la revisión realizada por Selwyn (2009), estas teorías describen a un estudiante con la capacidad de transformar los contextos escolares: "the internet-connected young learner is often celebrated as being no longer the passive recipient of educational instruction but instead cast into and active role of (re)constructing the nature, place, pace and timing of learning events as they wish" (367).

Estas miradas se condicen con lo que se espera en términos generales de la integración de NT al mundo educativo: apertura del contexto educativo a modelos menos jerárquicos, de corte constructivista y, por sobre todo, donde se incorpore la forma de aprender de la nueva generación de jóvenes (Fransen, 2006). Lo que se trata de hacer es de encontrar respuestas educativas innovadoras de modo que los nuevos aprendices se sientan más cómodos y se adapten de mejor manera en el contexto escolar (Pedró, 2006). Para estas teorías, el punto de referencia de esos cambios son los propios jóvenes, sus prácticas y opiniones. 


\subsection{NECESIDAD DE MAYOR INVESTIGACIÓN EMPÍRICA SOBRE LAS TNA}

En una línea más actual de estudios (e.g. Bennett, Maton y Kervin, 2008; Selwyn, 2009; Jones y Czerniewicz, 2010), se ha planteado que no se puede generalizar la TNA a todos los estudiantes, especialmente por falta de investigación empírica (Cabra-Torres y Marciales-Vivas, 2009). En general, estos autores postulan que la TNA se trataría de una sobresimplificación, ya que no considerarían las experiencias variadas que los jóvenes tienen con la tecnología.

Desde esta perspectiva se ha avanzado en identificar factores, como acceso a NT, género, nivel socioeconómico, características del entorno, que definirían distintos subgrupos en la generación jóvenes, descartando la edad (Helsper y Eynon, 2010) o la exposición a NT (Bennett y Maton, 2010) como únicas variables explicativas. Estas investigaciones concluyen principalmente que no existe un uso generalizado entre los jóvenes de distintas y variadas NT y herramientas web (Nasah et al., 2010; Thinyane, 2010), que no todos los jóvenes saben operar adecuadamente con la web o el computador (Hargittai, 2010) y que no existe evidencia para postular que los jóvenes adoptan estilos innovadores o diferentes de aprendizaje, prefiriendo incluso sistemas tradicionales (Margaryan, Littlejohn y Vojt, 2011). Otros investigadores, como Eynon y Malmberg (2011), han propuesto tipologías en relación al uso cotidiano de Internet, señalando que los distintos perfiles se explican por características individuales (como grupo etario y competencias tecnológicas) y factores contextuales (como acceso de NT en el hogar y regulación o no de padres en el uso de Internet).

En el contexto hispanohablante, nos encontramos con 2 textos que discuten la teoría de los nuevos aprendices. El primero corresponde a una revisión de investigaciones que critican los que algunos autores (Cassany y Ayala, 2008) denominan "la metáfora de los nativos digitales", encontrando que existe evidencia para postular que: los jóvenes no son conscientes de sus necesidades en información, por lo que no son autónomos para satisfacerlas; conocen la mecánica para acceder a Internet, pero no manejan conocimientos especializados; la navegación en Internet consiste más en ojear los sitios que en leerlos. El otro artículo encontrado se sitúa en el contexto chileno. Sánchez et al. (2010) realizan una investigación cualitativa a estudiantes de secundaria, concluyendo que la generación de estudiantes no cuenta con rasgos comunes, como multitasking o preferencia del lenguaje multimedia por sobre el escrito, y que los rasgos propuestos por las TNA sólo se darían en un grupo de estudiantes.

Este último estudio tiene un enfoque cualitativo, poco común en el estudio sobre jóvenes y tecnologías (Moyle y Owen, 2008). Como plantea Wijngaards (2009), los estudios que incorporan a los estudiantes se han centrado en el tipo de medios que usan y en la cantidad de tiempo que eso les toma, pero no se aborda el tema desde la experiencia con NT en ambientes de aprendizaje diversos, como serían la escuela y el hogar. No obstante, fue posible encontrar algunas investigaciones que incorporan la mirada de los estudiantes sobre el uso de NT en el contexto escolar y extra-escolar, que si bien no necesariamente incluyen la discusión sobre nuevos aprendices, sí aportan evidencia para responder la pregunta sobre la posibilidad de unir contextos y si acaso los jóvenes son conscientes de esa posibilidad. Por ejemplo, Kent y Facer (2004) estudian si existe esta vinculación desde lo que piensan estudiantes ingleses de $5^{\circ}, 7^{\circ}, 10^{\circ}$ y $12^{\circ}$ grado. Los autores señalan que los jóvenes quieren y van introduciendo lo que hacen en un 
contexto y otro (ejemplo: Power Point), concluyendo que los límites entre contextos son permeables en las percepciones de los estudiantes.

Sin embargo, si bien existe evidencia para sostener que los estudiantes son capaces de visualizar diferencias entre el uso que se les da a las NT en el hogar y en la escuela, no necesariamente proponen la necesidad de su interconexión ni maneras de lograrlo (Lohnes and Kinzer, 2007). Estudios como los de Sánchez et al. (2010) en Chile y el de Grant (2011) en Inglaterra demuestran que los jóvenes creen que es importante el uso de NT en sus vidas, pero perciben brechas importantes entre el contexto escolar y cotidiano. Selwyn, Potter y Cranmer (2009) muestran en un estudio etnográfico a alumnos de primaria que cuando se habla de conexión entre aprendizaje en el colegio y hogar, los entrevistados se centran en las tareas escolares; poco se trae del colegio a la casa y la experiencia escolar es muy diferente a lo que ocurre en sus hogares. En la revisión bibliográfica de Selwyn (2009), se señala que son variados los estudios que muestran que gran parte de los jóvenes esperan o incluso quieren que en sus escuelas se usen las NT como ellos lo hacen en sus hogares, pero no estarían tan deseosos de tecnologizar sus entornos como proponen las TNA. Un ejemplo de esto es el estudio de Spires et al. (2008), quienes demuestran que los jóvenes de su estudio (estadounidenses de $6^{\circ}$ a $8^{\circ}$ grado) hacen distinciones claras entre el uso que se da dentro y fuera de la escuela al computador, pero relacionan 'fuera de la escuela' con entretenimiento y comunicación, y 'dentro de la escuela' con aburrimiento, no obstante, prefieren usar las NT que no usarlas. De este modo, les gustaría trasladar su experiencia externa a la escuela, pero no son capaces de imaginarse cómo eso se podría lograr.

Una explicación a este último descubrimiento la propone Ben-David Kolikant (2010), quien en su investigación a estudiantes de $8^{\circ}$ a $11^{\circ}$ grado en Israel, concluye que es posible plantear que en una parte importante de estudiantes existe un sistema doble de valores (escolar y extra-escolar). Él encuentra que la mayoría de esos estudiantes perciben tener bajas competencias para el manejo de NT y capacidades de aprendizaje, incluso más bajas que generaciones anteriores, ya sea porque sus escuelas tienen una orientación tradicional de la enseñanza (del libro) o porque el uso de Internet en sí mismo simplifica las tareas que se realizan (incentiva la flojera); serían estudiantes que finalmente se alinean con los valores tradicionales de la escuela y asocian a esos valores el concepto de aprendizaje.

En definitiva, como postulan Bennett, Maton y Kervin (2008) y Selwyn (2009), son necesarios estudios en contexto y que incluyan percepciones sobre el uso de las NT entre los jóvenes, tanto para tener una buena línea base de sus experiencias, como para justificar cambios pertinentes en la educación a partir de esa caracterización y que no lleven a la aparición de nuevas brechas sociales. ${ }^{2}$ Asimismo, se rescata la importancia de incorporar datos cualitativos para comprender cómo los estudiantes entienden las NT (Margaryan, Littlejohn y Vojt, 2011). Por último, nosotros relevamos la necesidad de hacer mayor investigación interpretativa en países latinoamericanos y en contextos escolares.

2 Por ejemplo, que un sistema educativo innovador y tecnologizado deje fuera a quienes no ocupan las NT o no le gustan del modo que se esperaba (Thinyane, 2010). 


\section{METODOLOGÍA}

El objetivo de este estudio es caracterizar a jóvenes chilenos de educación secundaria a partir del uso y frecuencia que les dan a las NT y las percepciones que tienen sobre su contribución en contextos escolares y extra-escolares (hogar). En concreto, se espera poder responder las siguientes preguntas: ¿Cómo es el uso de NT que le dan los estudiantes en los dos contextos escuela y hogar? ¿Es posible plantear que los jóvenes estudiados corresponden a nuevos aprendices capaces de conectar ambos contextos?

Se trata de un estudio de pequeña escala exploratorio-descriptivo, que combina datos cuantitativos y cualitativos. La hipótesis de investigación es la siguiente: los estudiantes de esta investigación, que cuentan con la experiencia de usar NT tanto fuera como dentro del contexto escolar, serían capaces de establecer algunos vínculos entre el contexto escolar y el cotidiano.

\subsection{INSTRUMENTOS Y VARIABLES DE ESTUDIO}

Para conocer la frecuencia y tipo de uso de distintas NT entre los jóvenes estudiados, así como para conocer su evaluación de distintas contribuciones de ellas en distintos contextos, se utilizó un cuestionario autoaplicado. Para llegar a las percepciones e interpretaciones de los estudiantes sobre el uso de NT en distintos contextos, se aplicó una pauta de entrevista semi-estructurada a una sub-muestra de ellos.

Para la construcción de los instrumentos se definieron dos dimensiones de estudio: contexto escolar y contexto hogar, dentro de las cuales se definieron temáticas similares en las cuales indagar para poder hacer comparaciones y establecer posibles vínculos entre uno y otro contextos. La Tabla 1 muestra el esquema seguido para la construcción de instrumentos.

Tabla 1. Esquema de operacionalización para la construcción de instrumentos

\begin{tabular}{|c|c|c|c|}
\hline Dim & Sub-dimensiones & $\begin{array}{c}\text { Variables de estudio } \\
\text { encuestas }\end{array}$ & $\begin{array}{c}\text { Variables de estudio } \\
\text { entrevistas }\end{array}$ \\
\hline \multirow[t]{2}{*}{$\begin{array}{l}\text { Uso de NT en } \\
\text { el contexto } \\
\text { cotidiano } \\
\text { (hogar) }\end{array}$} & $\begin{array}{l}\text { Uso de NT } \\
\text { actividades } \\
\text { cotidianas }\end{array}$ & $\begin{array}{l}\text {-Frecuencia de uso de } \\
\text { distintas NT. } \\
\text {-Tipos de uso de NT } \\
\text {-Evaluación de } \\
\text { contribuciones del uso } \\
\text { de NT. }\end{array}$ & \multirow{3}{*}{$\begin{array}{c}\text {-Experiencia de uso } \\
\text { de NT. } \\
\text {-Contribuciones y } \\
\text { limitaciones del uso } \\
\text { de NT. } \\
\text {-Competencias que } \\
\text { se generan en el uso } \\
\text { de NT. } \\
\text {-Utilidad de lo que } \\
\text { se genera en el uso } \\
\text { de NT en otros } \\
\text { contextos. }\end{array}$} \\
\hline & $\begin{array}{c}\text { Uso de NT en tareas } \\
\text { escolares }\end{array}$ & $\begin{array}{l}\text {-Frecuencia de uso de } \\
\text { computador para tareas } \\
\text { escolar. } \\
\text {-Evaluación de } \\
\text { contribuciones del uso } \\
\text { de NT. }\end{array}$ & \\
\hline $\begin{array}{l}\text { Uso de NT en el } \\
\text { contexto escolar }\end{array}$ & $\begin{array}{c}\text { Uso de NT en } \\
\text { contexto escolar. }\end{array}$ & $\begin{array}{c}\text {-Evaluación de } \\
\text { contribuciones del uso } \\
\text { de NT. }\end{array}$ & \\
\hline
\end{tabular}


En el caso de las entrevistas, cuando se preguntó por contribuciones/limitaciones y utilidad del uso de NT en distintos contextos se hizo en referencia al aprendizaje, entendiéndolo como el proceso de adquisición conocimientos y competencias, que puede ocurrir en distintos contextos y en interacción con distintas personas y medios o herramientas.

Los instrumentos se centran en el uso del computador e Internet, por ser las NT comunes a los dos contextos estudiados. La aplicación de la encuesta duró entre 15 a 20 minutos por persona; y la de la pauta de entrevista, entre 30 minutos a 1 hora.

\subsection{SELECCIÓN DE MUESTRA Y TRABAJO DE TERRENO}

En primer lugar, se realizó el muestreo para la aplicación de la encuesta, el cual fue de carácter intencional no probabilístico al interior de tres establecimientos escolares que formaran parte de la red Enlaces del Ministerio de Educación, ${ }^{3}$ específicamente donde la sala de computación tuviera un uso curricular. Adicionalmente, los establecimientos contaron con otros requisitos: a) tener un SIMCE (Sistema de Medición de Calidad de la Educación) en $2^{\circ}$ medio en lenguaje y matemáticas superior al promedio nacional y regional; b) no ser establecimientos acomodados ni vulnerables (no tener un IVE, Índice Vulnerabilidad Escolar, de enseñanza media inferior al IVE Nacional de enseñanza media; y alumnos que no estuvieran clasificados ni en GSE "bajo" ni "alto", según clasificación SIMCE). Estos requisitos buscaron 'controlar' el contexto donde se da la experiencia escolar de uso de NT, evitando que en las entrevistas surgieran problemas asociados a malas condiciones económicas del establecimiento o a situaciones aisladas que sólo conocen los establecimientos más acomodados. El foco no está en establecer diferencias entre tipo de establecimientos, por eso mismo se eligen establecimientos similares; el foco está en conocer diferencias perceptuales y de opinión sobre distintos contextos de uso de NT.

Dentro de cada establecimiento, se encuestó a los estudiantes de tercer año medio ( $11^{\circ}$ grado). En total se encuestaron a 195 estudiantes de 16 a 18 años pertenecientes a 3 establecimientos particulares subvencionados de la ciudad de Santiago que cumplían con los requisitos antes señalados (Tabla 2).

Tabla 2. Muestra de estudiantes encuestados, según establecimientos

\begin{tabular}{|c|c|c|c|}
\hline & $\begin{array}{c}\text { Total } \\
\text { encuestado }\end{array}$ & $\begin{array}{c}\text { Total mujeres } \\
\text { encuestadas }\end{array}$ & $\begin{array}{c}\text { Total hombres } \\
\text { encuestados }\end{array}$ \\
\hline Establecimiento 1 & 61 & 61 & $0^{4}$ \\
\hline Establecimiento 2 & 29 & 9 & 20 \\
\hline Establecimiento 3 & 105 & 64 & 41 \\
\hline Total & 195 & 134 & 61 \\
\hline
\end{tabular}

\footnotetext{
3 El programa Enlaces del Ministerio de Educación de Chile tiene como objetivo trabajar con los aspectos que permiten la incorporación de las NT en contextos escolares subvencionados (municipales o privados): instalación de infraestructura tecnológica, desarrollo de contenidos y nuevos modelos pedagógicos, y desarrollo de competencias en profesores. Este programa corresponde a la experiencia de integración de computadores más extendida en nuestro país: un $95 \%$ de los estudiantes del sistema subvencionado de educación tienen acceso a NT en sus establecimientos escolares a través de este programa. Los establecimientos municipales y particulares subvencionados representan en conjunto más del $90 \%$ del total de establecimientos en Chile (Fuente: www.mineduc.cl).

4 Se trata de un establecimiento sólo de mujeres.
} 
Luego de aplicadas las encuestas, se entrevistó a 8 estudiantes ya encuestados, seleccionados intencionalmente por contar con la experiencia de uso de computador e Internet tanto en la escuela como en su casa. El factor de variabilidad estuvo dado por la frecuencia de uso cotidiano (diario) del computador (no se eligieron a estudiantes con menos de 1 hora de uso). La Tabla 3 muestra el detalle de la muestra seleccionada para las entrevistas.

Tabla 3. Muestra seleccionada y factores de variabilidad de entrevistas

\begin{tabular}{|c|c|c|}
\hline Clave & Género & $\begin{array}{c}\text { Horas de uso diario } \\
\text { de computador }\end{array}$ \\
\hline E1 & Femenino & $1 \mathrm{a} 4 \mathrm{hrs}$ \\
\hline E2 & Femenino & $1 \mathrm{a} 4 \mathrm{hrs}$ \\
\hline E3 & Femenino & $1 \mathrm{a} 4 \mathrm{hrs}$ \\
\hline E4 & Femenino & $1 \mathrm{a} 4 \mathrm{hrs}$ \\
\hline E5 & Femenino & más de $4 \mathrm{hrs}$ \\
\hline E6 & Masculino & más de $4 \mathrm{hrs}$ \\
\hline E7 & Masculino & más de 4 hrs \\
\hline E8 & Masculino & más de 4 hrs \\
\hline
\end{tabular}

Para procesar y analizar los datos cuantitativos se utilizó el software SPSS 17 y para los cualitativos se usó el software NVIVO 8. El tratamiento de ambos tipos de datos es de carácter descriptivo. En el caso de las entrevistas, durante el proceso de codificación se le dio espacio a nuevas categorías y sub-categorías que fueron surgiendo.

\section{RESULTADOS}

Los resultados se separan en experiencia de uso y el análisis de contribuciones y limitaciones del uso de NT. Cada temática, a su vez, se separa en los dos contextos estudiados.

\subsection{EXPERIENCIA DE USO}

\subsubsection{Experiencia de uso extra-escolar}

Como muestra la tabla 4, las NT más utilizadas por los estudiantes son el celular, el computador y el reproductor de música. 
Tabla 4. Frecuencia de uso cotidiano de... (\%)

\begin{tabular}{|c|c|c|c|}
\hline & Nunca & $\begin{array}{c}\text { Algunas veces al año } \\
\text { o al menos una vez } \\
\text { al mes }\end{array}$ & $\begin{array}{c}\text { Al menos una vez } \\
\text { por semana o Todos } \\
\text { los días }\end{array}$ \\
\hline Celular & 2,6 & 2,6 & 94,8 \\
\hline Computador & 1 & 6,2 & 92,7 \\
\hline Reproductor de música & 0,5 & 6,8 & 92,7 \\
\hline $\begin{array}{c}\text { Cámara digital de fotos y/o } \\
\text { video }\end{array}$ & 6,2 & 48,7 & 45,1 \\
\hline Consola de juegos & 35,8 & 38,4 & 25,9 \\
\hline
\end{tabular}

Respecto al uso cotidiano del computador, la mayor parte de la muestra $(61,1 \%)$ utiliza el computador diariamente entre 1 a 4 horas. Según el relato de las entrevistas, los estudiantes tienen usos muy similares del computador en sus casas: 'chatear' (messenger), uso de redes sociales (facebook, por sobre todo), escuchar música, descargar archivos, ver videos, jugar video juegos en computador o consola, hacer tareas y trabajos. Otros usos menos nombrados son: Photoshop e 'investigar en Internet' sobre temas que les interesan o bajar tutoriales para software. En resumen, dejando de lado las tareas escolares, se identifican dos usos principales: comunicarse y entretenerse y, en menor medida, aparece el aprendizaje de cosas nuevas. Consistente con las entrevistas, la encuesta muestra (Tabla 5) que el uso más frecuente entre los estudiantes es usar el computador para comunicarse con otros.

Tabla 5. Frecuencia de uso del computador para... (\%)

\begin{tabular}{|c|c|c|c|}
\hline & Nunca & $\begin{array}{c}\text { Algunas veces al año } \\
\text { o al menos una vez } \\
\text { al mes }\end{array}$ & $\begin{array}{c}\text { Al menos una vez } \\
\text { por semana o todos } \\
\text { los días }\end{array}$ \\
\hline $\begin{array}{c}\text { Comunicarse con otros (e-mail, } \\
\text { chat, foros, comunidades } \\
\text { virtuales) }\end{array}$ & 0 & 7,8 & 92,2 \\
\hline $\begin{array}{c}\text { Hacer tareas o trabajos para el } \\
\text { colegio }\end{array}$ & 1 & 13,5 & 85,4 \\
\hline $\begin{array}{c}\text { Para juegos o entretención } \\
\text { (intereses personales, música, } \\
\text { por ejemplo) }\end{array}$ & 20,7 & 35,7 & 43,5 \\
\hline
\end{tabular}

\subsubsection{EXPERIENCIA DE USO ESCOLAR}

La experiencia de uso de NT por parte de los estudiantes entrevistados se concentra en la sala de computación de los respectivos establecimientos para utilizar software de trabajo (Word, Excel, Power Point) o especializados (para una asignatura o para actividades 
específicas como hacer gráficos), mostrar videos o sitios web o, en algunos casos, para acceder a recursos educativos online. Los estudiantes señalan que la frecuencia de uso depende del profesor que dicte la clase (algunos les gusta más que a otros) y del tipo de contenido que se esté pasando y del tipo de actividad.

Los estudiantes no entregan muchos detalles al hablar de tipos de actividades que realizan con el computador. Todos reconocen su uso para reforzar materia o como parte de un trabajo y algunos señalan que se utiliza para investigar o buscar información. Algunos estudiantes son enfáticos al señalar que en la sala de computación no se pasa materia nueva. En esta línea, todos los estudiantes hacen distinciones claras entre la sala de computación y la sala de clases, donde las NT que se utilizan son el proyector, transparencias y presentación de videos/películas.

En la encuesta se les preguntó a los estudiantes por las asignaturas en las que usaban computador e Internet. Se usan mucho más en Lenguaje, Filosofía/Psicología, Ciencias e Historia y Geografía. Mucho menos común en Idioma extranjero (inglés), Matemáticas y Artes Visuales o Musicales.

\subsection{CONTRIBUCIONES Y LIMITACIONES DEL USO DE NT EN EL APRENDIZAJE}

\subsubsection{Contribuciones y limitaciones del uso cotidiano de NT}

En la encuesta (Gráfico 1) aparecen como más importante el uso del computador para: comunicación, tareas escolares y actividades de interés personal. Por otra parte, el computador sería menos importante para conocer nuevas personas, por lo que no reemplazaría las relaciones presenciales.

Gráfico 1. Porcentaje de estudiantes que responden 'importante' o 'muy importante' a ¿Qué tan importante es el uso de computador...?

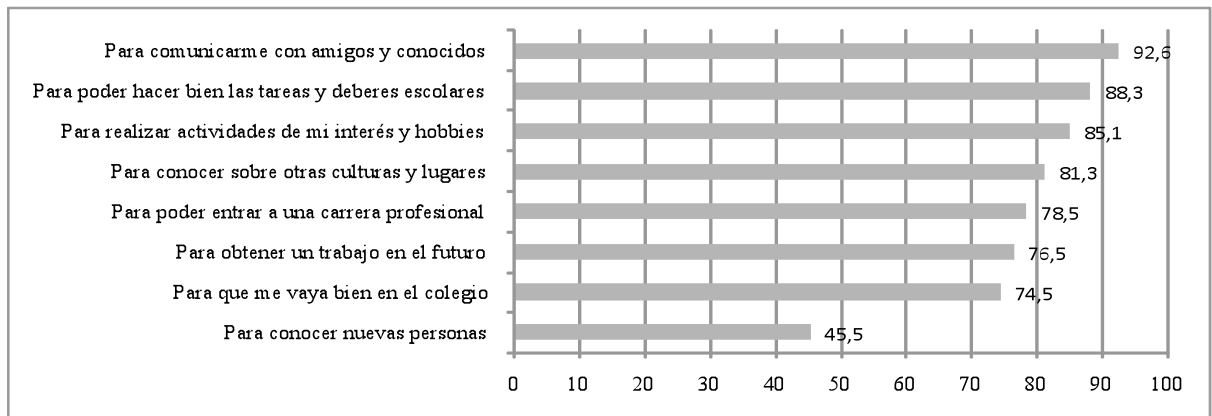

Las contribuciones que se mencionan en las entrevistas (Tabla 6) están relacionadas con la generación de habilidades computacionales en el uso del computador. Por lo indicado por los entrevistados, esas competencias no serían las mismas que se generan en actividades escolares, porque precisamente se utilizan otros recursos y herramientas. Asimismo, se menciona la posibilidad que entrega Internet para que los estudiantes busquen información sobre sus intereses personales. También se identifica, aunque no de manera generalizada, la contribución en la generación de espacios de comunicación, ya que para los estudiantes el computador facilita las relaciones sociales. 
Tabla 6. Contribuciones y limitaciones del uso de NT en el contexto extra-escolar para actividades cotidianas

(+) Competencias computacionales.

(+) Generación de espacios de comunicación.

(+) Conocimientos de interés personal de los estudiantes.

(-) Preocupación sobre relaciones sociales.

(-) Bajo aporte 'educativo' de las actividades extra-escolares en el computador.

En la encuesta se preguntó a los estudiantes cuáles competencias del listado de la Tabla 7 se desarrollan mejor con el uso cotidiano del computador. Las competencias más elegidas fueron Creatividad e Iniciativa y autonomía.

Tabla 7. ¿Cuál habilidad crees que se puede desarrollar mejor con el uso del PC que le das en tu casa u otro lugar que no sea el colegio?

\begin{tabular}{|c|c|}
\hline Creatividad & $33,3 \%$ \\
\hline Iniciativa y Autonomía & $27,9 \%$ \\
\hline Capacidad para manejar y seleccionar información & $16,4 \%$ \\
\hline Capacidad para solucionar problemas y conflictos & $10,3 \%$ \\
\hline Pensamiento Crítico & $5,5 \%$ \\
\hline Colaboración con otros y Trabajo en equipo & $4,2 \%$ \\
\hline Capacidad de liderazgo & $2,4 \%$ \\
\hline
\end{tabular}

En cuanto a las limitaciones de las NT al aprendizaje (Tabla 6), sólo se indagó en ellas en las entrevistas. Dos estudiantes muestran preocupación por las relaciones sociales que se generan a través de Internet, ya que alejaría del contacto presencial con otras personas. Sin embargo, lo que se menciona con especial énfasis en todos los entrevistados es el bajo 'aporte' educativo que tiene el uso extra-escolar del computador. Es más, lo ven como algo que desconcentra de sus responsabilidades. Incluso algunos son más tajantes al decir que dudan que en el uso cotidiano de NT se aprenda algo. (Refiriéndose al chat) “...no, como aprender no, porque más que nada lo ocupo para conversar, pero no como algo educativo" (E3). Dos alumnos señalan que para ellos se trata más que nada de una herramienta para conversar, que de aprendizaje. Es posible afirmar que en el relato de los entrevistados se disocia aprendizaje y entretención y, aprendizaje y contexto extraescolar. "No, tengo más comunicación, pero no creo que aprenda mucho, aprendo más aquí en el colegio que en la casa" (E2).

Muy relacionado con lo anterior, la mayoría de los entrevistados no le ve utilidad para tareas escolares al uso que le dan al computador en sus casas. La excepción la tendrían las habilidades computacionales, especialmente saber ocupar software para realizar mejores 
trabajos. Sin embargo, al mismo tiempo los estudiantes que mencionan esto perciben que se debe a su propio interés en las tecnologías. Por otro lado, un par de estudiantes mencionan que los software que usan por interés propio no son los mismos que los del colegio. Al preguntarles por la utilidad de otros aprendizajes para el mundo escolar, hay estudiantes que de forma enfática señalan que no le ven utilidad para el colegio al uso que le dan al computador en sus casas. "O sea, quizás uno aprende a chatear y a, no sé, quizás a tratar de... concentrarse a la vez, pero algo así como que me sirva en el colegio, no creo" (E7).

\subsubsection{Contribuciones y limitaciones del uso de $N T$ \\ en tareas escolares en el contexto extra-escolar}

En la encuesta (Tabla 8) aparece que lo más valorado del uso del computador en tareas escolares es que se pueden realizar otras actividades al mismo tiempo, seguido por la posibilidad de mejorar habilidades computacionales. Con alta aprobación, pero más lejos, se encuentra la contribución de entender la materia mejor que en el colegio.

Tabla 8. Grado de acuerdo con contribuciones de las NT en el contexto extra-escolar en tareas escolares ${ }^{5}$

\begin{tabular}{|c|c|c|}
\hline $\begin{array}{c}\text { Cuando haces tareas escolares con el } \\
\text { computador fuera del colegio.... }\end{array}$ & $\begin{array}{c}\% \text { de acuerdo y } \\
\text { muy de acuerdo }\end{array}$ & $\begin{array}{c}\% \text { en desacuerdo } \\
\text { y muy en } \\
\text { desacuerdo }\end{array}$ \\
\hline $\begin{array}{c}\text { Al mismo tiempo puedo hacer otras cosas en } \\
\text { el PC (Juegos, chat, e-mail, otros) }\end{array}$ & 93,7 & 6,3 \\
\hline $\begin{array}{c}\text { Mejoro mis habilidades para usar el } \\
\text { computador }\end{array}$ & 88,5 & 11,5 \\
\hline Entiendo mejor la materia que en el colegio & 70,5 & 29,5 \\
\hline
\end{tabular}

En las entrevistas, respecto al uso del computador para tareas escolares (Tabla 9), los estudiantes sólo identifican contribuciones. En primer lugar, todos señalan que el computador es un apoyo en la realización de tareas, ya que facilita y agiliza esta labor, en comparación a la utilización de libros. Todos también señalan que el uso de Internet es fundamental para llevar a cabo las tareas, especialmente por la cantidad de información disponible, facilitando la búsqueda de información. Valoran, asimismo, el contexto del hogar especialmente porque no hay controles ni de tiempo ni desde el profesor. "Igual es que como que cuando estoy sola, como que me concentro más, leo más, como que aparte no tengo la presión de que van a tocar y que tengo que terminar el trabajo rápido" (E4). Finalmente, los estudiantes consideran que es ventajoso usar el computador en tareas escolares en la casa porque permite resolver dudas con compañeros que están conectados en red a través de mensajería instantánea. “...busco toda la información, si no entiendo algo, me meto a Internet, sino entiendo una tarea y me la piden, me meto a

5 Originalmente había ítems en negativo. Para la presentación de la información se cambiaron todos a afirmativo.

Esto se hizo para todas las preguntas tipo escala. 
Facebook, a Messenger, o a lo que sea, o facilito o me facilitan información" (E5). En términos generales, los estudiantes creen que son capaces de hacer sus tareas y estudiar por su cuenta. No obstante, la mitad de los estudiantes señalan que, en último término, requieren de la aprobación final del profesor. Recordar que en la Tabla 8, 'entiendo mejor la materia que en el colegio' era la contribución con menos aprobación.

Tabla 9. Contribuciones y limitaciones del uso de NT en el contexto extra-escolar para tareas escolares (entrevistas)

(+) Preferencia del contexto extra-escolar (los profesores opinan lo contrario)

(+) Apoyo en la realización de tareas

(+) Resolución de dudas entre compañeros.

\subsubsection{Contribuciones y limitaciones de las NT en el contexto escolar}

Como se observa en la Tabla 10, la contribución más valorada por los estudiantes dice relación con una mayor comprensión de la materia. Le siguen de cerca, la entretención que produce el uso de NT, la posibilidad de aportar con ideas y opiniones propias y el sentimiento compartido de mayor motivación al trabajar con NT. Donde se evidencia una leve tendencia negativa es en el grado de acuerdo con la existencia de cambios en la relación de los profesores y los alumnos a una relación más cercana (48,2\% de aprobación).

Tabla 10. Grado de acuerdo con contribuciones de las NT en el contexto escolar

\begin{tabular}{|c|c|c|}
\hline En las clases donde se ocupan tecnologías... & $\begin{array}{c}\% \text { de } \\
\text { acuerdo } \\
\text { y muy de } \\
\text { acuerdo }\end{array}$ & $\begin{array}{c}\% \text { en } \\
\text { desacuerdo } \\
\text { y muy en } \\
\text { desacuerdo }\end{array}$ \\
\hline Entiendo mejor la materia & $87,2 \%$ & $12,8 \%$ \\
\hline Me entretengo & $85,1 \%$ & $14,9 \%$ \\
\hline Puedo aportar con mis ideas y opiniones & $85,1 \%$ & $14,9 \%$ \\
\hline Yo y mis compañeros estamos más motivados & $84,5 \%$ & $15,5 \%$ \\
\hline Es fácil ayudarnos y colaborar con mis compañeros & $78,4 \%$ & $21,6 \%$ \\
\hline Mejoro mis habilidades para usar el computador & $76,4 \%$ & $23,6 \%$ \\
\hline Hago las actividades más rápido & $74,4 \%$ & $25,6 \%$ \\
\hline No me cuesta concentrarme & $69,2 \%$ & $30,8 \%$ \\
\hline $\begin{array}{c}\text { Me es más fácil ver en qué me equivoco y corregir } \\
\text { errores }\end{array}$ & $64,1 \%$ & $35,9 \%$ \\
\hline $\begin{array}{c}\text { Yo y mis compañeros ponernos atención sin ponernos } \\
\text { a hacer otras cosas }\end{array}$ & $62,1 \%$ & $37,9 \%$ \\
\hline $\begin{array}{c}\text { Mi profesor es más cercano conmigo y mis } \\
\text { compañeros (proxy de cambios en la sala) }\end{array}$ & $48,2 \%$ & $51,8 \%$ \\
\hline
\end{tabular}


En las entrevistas, el análisis de las contribuciones y limitaciones del computador en el contexto escolar arrojó una serie de aspectos positivos y negativos que se presentan en la Tabla 11 y que se condicen con la visión de la tabla anterior (Tabla 10).

Tabla 11. Contribuciones y limitaciones del uso de NT en el contexto escolar (entrevistas)

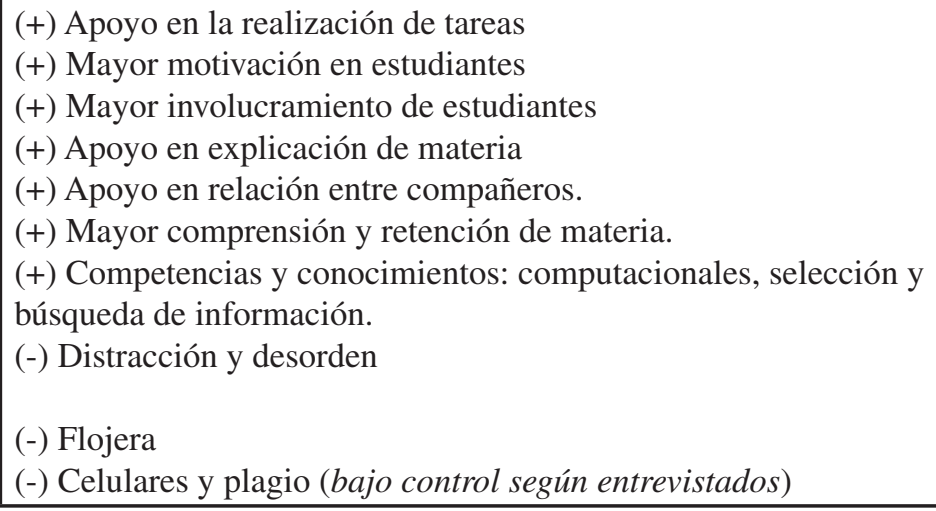

Al igual que para actividades escolares extra-escuela, las contribuciones identificadas se concentran en el apoyo para la realización de tareas: se realizan con mayor rapidez, requieren de menos esfuerzo e Internet pone a disposición de información. Se menciona también como contribución la mayor motivación que genera el uso de computador en las clases, porque permitiría clases "más relajadas y más entretenidas" y con la posibilidad hacer actividades extra-académicas (chat, facebook, navegación). "Igual es más entretenido. Es que hay que salir de lo común porque estar aquí sentada en la sala es fome. (...) la sala de computación es más cómoda, es más grande y... en el computador es más entretenido, uno puede hacer más cosas que mirando el Data" (E2). También valoran la posibilidad que entrega el computador de involucrar más a los estudiantes en las tareas escolares, visto esto como mayor participación, un trabajo más independiente y la posibilidad de ponerle un "sello personal" a lo que están haciendo. Otra contribución es el apoyo en la explicación de la materia que se relaciona con la posibilidad que entregan las NT para visualizar ideas y para ejemplificar a partir de imágenes. Por último, el apoyo en la relación con los compañeros se refiere a la mayor interacción que se genera en la sala de computación entre los alumnos, eso sí, no a través de las NT (por ej., chat) sino porque no todos los estudiantes tienen las competencias tecnológicas necesarias, por lo que necesitan ayuda de sus pares y/o porque el profesor no puede ayudar a todos personalmente.

Por último, aparecen contribuciones relacionadas con los resultados que puede conllevar trabajar con las NT, como son: mayor retención y comprensión de la materia y la generación de habilidades tecnológicas y competencias de selección y búsqueda de información. Esto se condice con lo que aparece en los resultados de la encuesta, donde esta competencia es la más seleccionada por los jóvenes encuestados (Tabla 12), aunque también más de un quinto menciona Creatividad $(21,6 \%)$. En el contexto hogar, Creatividad ocupaba el segundo lugar, mientras la capacidad para manejar y seleccionar información, el tercero. 
Tabla 12. ¿Cuál habilidad crees que se puede desarrollar mejor con el uso del PC que le das en clases?

\begin{tabular}{|c|c|}
\hline Capacidad para manejar y seleccionar información & $38,9 \%$ \\
\hline Creatividad & $21,6 \%$ \\
\hline Colaboración con otros y Trabajo en equipo & $16,2 \%$ \\
\hline Iniciativa y Autonomía & $10,2 \%$ \\
\hline Capacidad para solucionar problemas y conflictos & $9,6 \%$ \\
\hline Pensamiento Crítico & $2,4 \%$ \\
\hline Capacidad de liderazgo & $1,2 \%$ \\
\hline
\end{tabular}

En cuanto a las limitaciones (Tabla 11), éstas se relacionan con el potencial de las NT de sacar a los estudiantes del foco de la clase que es la materia. Lo más nombrado por los entrevistados son la distracción y desorden que generaría el uso de computador y en la mitad de los relatos aparece la flojera: "...El computador es mucho más rápido, pero te enseña a ser mucho más flojo, como que uno podría hacer todo sentado en el computador. En cambio, igual los libros sirven de algo... uno lee más, buscando a mano, lee más, no solo lo que necesitas" (E4). Por último, se preguntó explícitamente por el tema del plagio y del uso de celulares. Los estudiantes dicen que el plagio es algo controlado, aunque los estudiantes mencionan que tienen "técnicas" para que los profesores no se den cuenta. Sobre los celulares, los entrevistados consideran su uso algo distractivo para la clase. El tema de la distracción que produce el uso de computador se condice con las respuestas de encuesta, donde no es tan marcada la aprobación a la afirmación de que los estudiantes no se distraigan con otras cosas (tabla 10).

Por otra parte, la mayoría de los estudiantes entrevistados indica que le ve a la materia vista en clases poca o casi ninguna utilidad para su vida cotidiana, no así para el contexto escolar. Cuando se les pregunta por algún tipo de aprendizaje que nace del uso de NT que sientan que les sirve para su vida extra-escolar, la mitad señalan que las habilidades computacionales les son útiles, señalando que uno de los usos es ayudar a miembros de su familia, principalmente a sus padres. "Hay cosas que nos enseñan que sí son para el colegio y de ahí no salen, pero por ejemplo, Excel, te ayuda en la casa porque, para el presupuesto de la casa, la mercadería y todo eso" (E5). Un sólo estudiante señala que las habilidades computacionales son muy útiles para aplicarlas en las asignaturas del colegio.

\section{CONCLUSIONES}

De los resultados presentados, se extraen 3 conclusiones que se exponen a continuación:

\subsection{ESTUDIANTES CUENTAN SÓLO CON ALGUNOS RASGOS DE LOS NUEVOS APRENDICES}

Es posible plantear que los jóvenes estudiados cuentan con algunos rasgos propuestos por las TNA en cuanto a su uso cotidiano de NT. ${ }^{6}$ En primer lugar, la encuesta muestra 
que los estudiantes usan NT variadas, como celular, computador y reproductores de música. Asimismo, la mayoría de la muestra tiene un uso diario del computador que va de 1 a 4 horas. En segundo lugar, las entrevistas muestran que respecto a los tipos de uso que se les da al computador, se trata de actividades variadas que dicen relación con la comunicación, entretención y realización de tareas escolares. Las entrevistas también muestran algunas prácticas colaborativas entre los jóvenes para la realización de tareas en sus casas cuando se usa el computador y preferencias por el autoaprendizaje, así como preferencias en el uso de Internet por sobre el libro como fuente de información. Por último, los estudiantes encuestados y entrevistados tienen una visión muy positiva sobre el uso de NT en general y son capaces de ver diferencias importantes entre ellos, valorando e interesándoles más el uso que le dan en sus hogares que en sus escuelas. Asimismo, a partir del relato de los entrevistados, es posible concluir que las mayores contribuciones del uso del computador e Internet en actividades escolares se encuentran en el proceso de enseñanza-aprendizaje y menos en los resultados, como son las contribuciones de apoyar la realización de tareas, mejorar comprensión de contenidos, motivar, entre otros.

Sin embargo, en los resultados aparecen algunos matices que nos impiden concluir de forma certera que los estudiantes de nuestra muestra correspondan a una nueva generación de aprendices. En primer lugar, aunque el uso de NT no se concentre en una sola herramienta, el uso de consolas y videojuegos no es generalizado como se podía concluir de la propuesta de TNA. Asimismo, la valoración de las NT no llega al punto de reemplazar las relaciones presenciales. En último lugar, los estudiantes si bien ven diferencias entre contextos, no proponen que el contexto escolar se abra para adoptar la forma en que ellos utilizan las NT en sus hogares para realizar tareas escolares y menos para comunicarse o entretenerse, como se verá con más detalle en las conclusiones siguientes.

\subsection{BRECHA ENTRE CONTEXTOS Y POCAS POSIBILIDADES DE VINCULACIÓN}

Como se señaló, los estudiantes establecen importantes diferencias entre los contextos estudiados. De la encuesta, es importante mencionar las diferencias en relación a las competencias que se pueden generar en el uso de NT en uno u otro contexto. En el hogar se estarían formando competencias de creatividad, iniciativa y autonomía; mientras en la escuela, la capacidad de manejar y seleccionar información, en segundo lugar, creatividad y, en tercero, colaboración con otros.

En las entrevistas también se visualiza esta brecha entre contextos. En primer lugar, al igual que en el estudio de Spires et al. (2008), los estudiantes relacionan el uso extra-escuela con comunicación y entretención y el intra-escuela con aprendizaje o, por contraposición, con deber y concentración. El uso cotidiano del computador no se corresponde con su uso educativo. La brecha se produce cuando parecen cerrarse las puertas para innovar y para insertar habilidades que provienen del uso cotidiano de NT en el contexto escolar, como la forma de colaboración a través de herramientas comunicacionales que cuentan los estudiantes que se produce cuando necesitan ayuda en sus casas y que no se da en el colegio porque están prohibidos los chats.

6 Se hace difícil distinguir qué características de la experiencia de uso intra-escuela estarían relacionadas con las TNA, ya que éstas no definen cómo un estudiante de la nueva generación de aprendices se comporta dentro de su establecimiento escolar, como si lo hacen respecto a sus prácticas cotidianas. 
Cuando se revisan las opiniones de los estudiantes sobre la utilidad del uso del computador en los dos contextos estudiados, lo único que pareciera moverse de un lugar a otro son las competencias computacionales. Incluso algunos estudiantes señalan que las competencias computacionales adquiridas en un contexto no necesariamente sirven en el otro. Cualquier otra práctica o aprendizaje no les parece que sea útil en el otro contexto. Los estudiante no son conscientes de cómo se podría lograr esa unión, más aun, no manifiestan que quieran unir los dos contextos.

Según las teorías de los nativos digitales, serán los propios estudiantes los que mostrarán las conexiones entre los diversos usos que les dan a las NT. Sin embargo, en los resultados de esta investigación parece difícil concluir esto. Desde los postulados de Ben-David Kolikant (2010), es plausible plantear que los estudiantes conviven con dos sistemas valóricos (escuela y hogar) que no han sabido articular.

\subsection{CONCEPTO ESCOLARIZADO DE APRENDIZAJE}

Una posible explicación a la imposibilidad de unir los dos contextos estudiados desde las percepciones y opiniones de los estudiantes, es que en ellos existe una visión escolarizada de lo que es el aprendizaje.

En las TNA, serían los profesores o inmigrantes digitales quienes tendrían una mirada escolarizada del aprendizaje y no los estudiantes. Sin embargo, en este estudio se encuentra esa mirada en los estudiantes. Hay algunos resultados que nos orientan a esta conclusión en el contexto escolar: a) los estudiantes ponen énfasis en la entretención, pero esa entretención no es asumido como aprendizaje. Lo que es distracción en el contexto escolar, es entretención en el extra-escolar; b) las contribuciones de las NT en el contexto escolar y tareas escolares, se centran en valorar prácticas tradicionales de enseñanza-aprendizaje. Las NT serían un apoyo, no un cambio en la forma de hacer la clase, donde éstas son beneficiosas como medios que ofrecen reducción de trabajo y disminución de incertidumbre (visión utilitaria); c) los perjuicios mencionados por los entrevistados tienen que ver con el potencial que tiene el uso de tecnologías para desviar la atención de los estudiantes de las actividades y actitudes académicas: distracción, desorden y flojera.

También refuerzan esta conclusión resultados encontrados en el contexto extra-escolar. Tenemos, por una parte, que si bien los estudiantes valoran el contexto extra-escolar por la mayor libertad para la realización de tareas, por la presencia de compañeros e Internet para buscar información, finalmente los estudiantes mencionan que quien aprueba o no lo que se hace en el hogar es el profesor, en otras palabras, se recurre finalmente a la validación escolar. En cuanto a las tareas extra-escolares, sólo basta recordar el bajo aporte educativo que le veían los entrevistados a estas actividades.

En las actividades cotidianas con el computador, ellos no ven que se generen instancias de lo que ellos entienden por aprendizaje escolar. Podríamos hipotetizar que en realidad lo que sucede es que no tienen conciencia de algunos aspectos, por ejemplo, de cómo usan las redes sociales para hacer tareas y de cómo colaboran entre ellos para esto. No se trata de no valorar el uso de NT, de hecho, este mismo análisis ha dado cuenta que los entrevistados identifican variadas contribuciones, pero cuando se trata del concepto de 'aprendizaje' pareciera existir una disociación entre los distintos contextos y usos. 


\section{DISCUSIÓN}

A diferencia de la hipótesis planteada, los estudiantes de esta investigación son pocas las vinculaciones que establecen entre el contexto escolar y extra-escolar en relación al uso de NT. Podríamos señalar que el grupo estudiado, que en cuanto a condiciones de muestreo y a percepciones y opiniones es bastante homogéneo, no se condice con la propuesta de la TNA sobre el nivel de conciencia de las posibilidades de vinculación que existirían entre la escuela y el hogar.

Es posible plantear que existe una baja reflexividad y la existencia de percepciones arraigadas sobre lo que significa la educación, aprendizaje, herramientas pedagógicas e incluso medios de comunicación y su lugar en la sociedad. Se hace difícil plantear la existencia de nativos digitales o nuevos aprendices si en ellos no hay una propuesta al menos encaminada sobre lo que sucede en distintos contextos de aprendizaje. En esta situación, se puede proponer que sea la escuela un agente de cambio y no sólo los estudiantes.

Ben-David Kolikant (2010) propone que es la escuela un agente clave para resolver las tensiones entre contextos con valores diferentes. No se trataría de que la escuela se adaptara totalmente a los valores de los estudiantes o viceversa, sino que la escuela explicitara a los estudiantes estos dos sistemas valóricos y presentarles sus ventajas y desventajas, ayudando a los estudiantes a usar selectiva y conscientemente valores y prácticas diferentes que a veces pueden ser incluso contradictorias (2010: 1390). En la misma línea, Bennett y Maton (2010) plantean precisamente que un error de la TNA es desvalorizar a la escuela y su posibilidad de cambio. Hay que valorizar la educación formal como un complemento a la informal (contexto hogar), no se trata de un llamado a que la primera se abra tanto que se fusione con la segunda.

Por último, aunque esta investigación es de corto alcance, por su carácter exploratoriodescriptivo, nos abre a la posibilidad de plantear nuevos caminos por los cuales transitar empíricamente. En primer lugar, resulta de importancia abrir la investigación y discusión a nuevos contextos y momentos. En los relatos de los estudiantes, aparece la distinción no sólo entre escuela y hogar, sino también dentro del colegio, entre sala de clases y sala de computación, y entre asignaturas según el tipo de profesor; y dentro del hogar, entre tareas escolares y tareas cotidianas. Por otra parte, aunque sólo se preguntó en las encuestas, aparece el momento futuro como otro espacio en cual se pueden estudiar expectativas y propuestas de los estudiantes, ya que aparece en los estudiantes una alta valoración de las NT para el trabajo y estudios futuros. Sería interesante incluso indagar en percepciones en esos dos contextos. En segundo lugar, destacar la importancia de la investigación interpretativa, de carácter cualitativo (entrevistas o grupos focales) en el estudio de jóvenes y nuevos aprendices, ya que es posible plantear que las encuestas llevan a respuestas más positivas y sin matices que las que se encuentran en las entrevistas. Por otra parte, la encuesta entrega de antemano las respuestas, sin dejar espacios para nuevas contribuciones, limitaciones o para observar el nivel de reflexividad de los actores estudiados. Incluso es posible visualizar que sólo con los datos cuantitativos se podría llegar a concluir que se está en presencia de nativos digitales, mientras que con las percepciones recogidas con las entrevistas no.

En definitiva, estos resultados refuerzan la propuesta de seguir investigando en países en vías de desarrollo o no desarrollados, incorporando la pregunta por las experiencias 
de los jóvenes en distintos contextos, especialmente si se está discutiendo la importancia y necesidad de hacer cambios en los sistemas educativos.

\section{REFERENCIAS BIBLIOGRÁFICAS}

Ben-David Kolikant, Y. (2010). Digital natives, better learners? Students' beliefs about how the Internet influenced their ability to learn. Computers in Human Behavior, vol. 26, 1384-1391.

Bennett, S. \& Maton, K. (2010). Beyond the 'digital natives' debate: Towards a more nuanced understanding of students' technology experiences. Journal of Computer Assisted Learning, vol. 26, 321-331.

Bennett, S., Maton, K.\& Kervin, L. (2008). The 'digital natives' debate: A critical review of the evidence. British Journal of Educational Technology, vol. 39, n. 5, 775-786.

Cabra-Torres, F. y Marciales-Vivas, G. (2009). Mitos, realidades y preguntas de investigación sobre los 'nativos digitales': una revisión. Universitas Psychologica, vol. 8, n. 2, 322-338.

Cassany, D. y Ayala, G. (2008). Nativos e inmigrantes digitales en la escuela. CEE Participación Educativa, vol. 9, 53-71.

Eynon, R. \& Malmberg, L. (2011). A typology of young people's Internet use: Implications for education. Computers \& Educaiton, vol. 56, 585-595.

Ferrés, J. (2000). Educar en una cultura del espectáculo. Barcelona: Paidós.

Fransen, J. (2006). Eennieuwewerkdefinitie van blended learning (A New Working Definition of Blended Learning). Dutch Open University: Journal 'OnderwijsInnovatie', vol. 8, n. 2, 26-29.

Grant, L. (2011). 'I'm completely different person at home': using digital technologies to connect learning between home and school. Journal of Computer Assisted Learning, vol. 27, 292 -302.

Hargittai, E. (2010). Digital Na(t)ives? Variation in Internet Skills and Uses among Members of the "Net Generation". Sociological Inquiry, vol. 80, n. 1, 92-113.

Helsper, E. \& Eynon, R. (2010). Digital natives: where is the evidence? British educational research journal, vol. 36, n. 3, 503-520.

Índice Generación Digital (IGD) (2009). Estudio Índice Generación Digital 2004-2008. Educarchile, Chile. Web. http://www.educarchile.cl/Portal.Base/Web/verContenido.aspx?ID=195398 [01/06/12]

International Telecommunication Union (ITU). (2008). Use of Information and Communication Technology by the World's Children and Youth. A statistical compilation. Ginebra: Places de Nations.

International Telecommunication Union (ITU). (2010). Medición de la sociedad de la información 2010. Ginebra: Places de Nations.

Jones, C. \& Czerniewicz, L. (2010). Editorial: Describing or debunking? The net generation and digital natives. Journal of Computer Assisted Learning, vol. 26, 317-320.

Kent, N. \& Facer, K. (2004). Different worlds? A comparison of young people's home and school ICT use. Journal of Computer Assisted Learning, vol. 20, 440-455.

Lohnes, S. \& Kinzer, C. (2007). Questioning assumptions about students' expectations for technology in college classrooms. Innovate: Journal of Online Education, vol. 3. http://www.innovateonline.info/ pdf/vol3_issue5/Questioning_Assumptions_About_Students\%27_Expectations_for_Technology_ in_College_Classrooms.pdf [03/06/12]

Margaryan, A., Littlejohn, A. \& Vojt, G. (2011). Are digital natives a myth or reality? University students' use of digital technologies. Computers \& Education, vol. 56, 429-440.

Martín Barbero, J. (1996). Heredando el futuro. Pensar la educación desde la comunicación. $C \& E$ (Cultura y Educación), vol. 9, 17. 
Moyle, K. \& Owen, S. (2008). Students' Voices Learning with Technologies. Students' expectations about learning with Technologies: A literature review. Australia: University of Canberra.

Nasah, A., DaCosta, B., Kinsell, C. \& Seok, S. (2010). The digital literacy debate: an investigation of digital propensity and information and communication technology. Education Tech Research Dev, vol. 58, 531-555.

Oblinger, D. \& Oblinger, J. (Eds.) (2005). Educating the Net Generation. USA: EDUCAUSE.

Pedró, F. (2006). Aprender en el nuevo milenio. Un desafío en nuestra visión de las tecnologías y la enseñanza. Paris: OECD.

Prensky, M. (2001a). Digital natives, digital immigrants. On the Horizon, vol. 9, n. 5, s/p.

Prensky, M. (2001b). Digital natives, digital immigrants, part II. Do they really think differently? On the Horizon, vol. 9, n. 6, s/p.

Prensky, M. (2006). Don't bother me mom I'm Learning. St. Paul, MN: Paragon House Publishers.

Prensky, M. (2007). How to teach with technology: keeping bother teachers and students comfortable in an era of exponential change. Emerging Technologies for Learning, vol. 2.

Sánchez, J., Salinas, A., Contreras, D. y Meyer, E. (2010). Does the new digital generation of learners exist? A Qualitative Study. British Journal of Educational Technology, vol. 42, n. 4, 543-556.

Selwyn, N. (2009). The digital native - myth and reality. New Information Perspectives, vol. 61, n.4, 364-379.

Selwyn, N., Potter, J. \& Cranmer, S. (2009). Primary pupils' use of information and communication technologies at school and home. British Journal of Educational Technology, vol. 40, n. 5, 919-932.

Spires, H., Lee, J., Turner, K. \& Johnson, J. (2008). Having Our Say: Middle Grade Student Perspectives on School, Technologies, and Academic Engagement. Journal of Research on Technology in Education, vol. 40, n. 4, 497-515.

Tapscott, D. (1998). Creciendo en un entorno digital: la generación net. Santafé de Bogotá: McGraw-Hill.

Thinyane, H. (2010). Are digital natives a world-wide phenomenon? An investigation into South African first year students' use and experience with technology. Computers \& Education, vol. 55, 406-414.

Wijngaards, G. (2009). Students' Voices research Young people's views on ICT. Web: http:// www.inholland.n1/NR/rdonlyres/B7E53AD1-D05A-4AFC-AE8B-4EEF1D5B28BC/12753/ MicrosoftWordStudents VoicesEnglisharticle1.pdf [01/06/12]. 